KCL-MTH-04-04

hep-th/0404062

\title{
$N=2$ Superconformal boundary states for free bosons and fermions
}

\author{
Matthias R. Gaberdiel* \\ Institut für Theoretische Physik \\ ETH Zürich \\ CH-8093 Zürich \\ Switzerland \\ and \\ Hanno Klemm ${ }^{\dagger}$ \\ Department of Mathematics \\ King's College London \\ Strand \\ London WC2R 2LS \\ $U K$ \\ and \\ Institut für Theoretische Physik \\ ETH Zürich \\ CH-8093 Zürich \\ Switzerland
}

\begin{abstract}
The most general $N=2$ superconformal boundary states for the $c=3$ theory consisting of two (uncompactified) free bosons and fermions are constructed. It is shown that the only $N=2$ boundary states are the familiar Dirichlet boundary states, as well as the Neumann boundary states with an arbitrary electric field.
\end{abstract}

${ }^{*}$ mrg@phys.ethz.ch

${ }^{\dagger}$ klemm@phys.ethz.ch 


\section{Introduction}

Most D-branes that have been considered in string theory so far are rather special in the sense that they preserve more symmetries than is actually required for their consistency. For example, for the case of string theory in flat space, the usual Neumann and Dirichlet branes preserve all the $\mathrm{U}(1)$ current symmetries associated to the spacetime coordinates. In terms of their boundary states, this means that they satisfy the gluing conditions

$$
\left.\left.\left(\alpha_{n}^{i} \pm \tilde{\alpha}_{-n}^{i}\right) \| B\right\rangle\right\rangle=0,
$$

where the $+(-)$ sign applies if $i$ is a Neumann (Dirichlet) direction. However, as was already pointed out by Polchinski [1], the consistency of the construction only requires that the boundary state preserves the conformal symmetry

$$
\left.\left.\left(L_{n}-\tilde{L}_{-n}\right) \| B\right\rangle\right\rangle=0 .
$$

Since $L_{n}$ is quadratic in the $\alpha$ 's, equation (1.1) implies equation (1.2) but the converse is not true. It is therefore interesting to determine the additional boundary states that only preserve the conformal symmetry (1.2), but not (1.1).

For the case of the theory consisting of a single free boson, this question was answered in $[2-6]$, and the generalisation to the $N=1$ supersymmetric theory consisting of a free boson and a free fermion was considered in [4]. While there are, in both cases, new D-branes other than the usual Neumann and Dirichlet branes, none of the additional branes are stable. In fact none of these branes preserves spacetime supersymmetry, since spacetime supersymmetry requires an unbroken $N=2$ worldsheet supersymmetry [7], which was not present in those theories. It is therefore of particular importance to study the above question in the context of an $N=2$ worldsheet theory. The simplest example of such a theory is the theory of two free bosons and fermions with $c=3$, and this is the theory we shall be analysing in this paper. As we shall show, in this case all $N=2$ superconformal D-branes can be accounted for in terms of the usual Neumann and Dirichlet branes. This is quite a surprising result since one may have thought by analogy with the situation for $N=1$ supersymmetry that there should be many more $N=2$ D-branes. On the other hand, our result is quite reassuring since it implies that, at least in this example, the usual Dirichlet and Neumann branes already account for all spacetime supersymmetric branes. One expects that there are other backgrounds for which one should be able to find 'new' $N=2$ D-branes; we believe that the techniques that are described in this paper will help to construct these D-branes.

D-branes that preserve at least the $N=2$ supersymmetry have been studied before in the framework of Gepner models and Calabi-Yau compactifications (see for example [8,9]), for $N=2$ Landau-Ginzburg models [10] and non-linear sigma models (see for example [10-12]) and for $N=2$ minimal models and coset models [13-16]. More recently $N=2$ D-branes have also been analysed in Liouville theory [17-19]. In most cases however, the D-branes that have been considered preserve more symmetry than just the $N=2$ algebra. Obviously, for $N=2$ minimal models all $N=2 \mathrm{D}$-branes are known, but this is simply a consequence of the fact that the whole theory is rational with respect to this algebra; in our example, the theory is not rational with respect to the $N=2$ algebra, and it is therefore a priori not obvious what the most general $N=2$ D-branes should be.

The paper is organised as follows. In section 2 we review some relevant facts about the $N=$ 2 superconformal algebra and its free field realisation. The Neveu-Schwarz boundary states 
are constructed in section 3, and in section 4 we consider the cylinder amplitude of two such boundary states. These amplitudes agree with the amplitudes of the usual Neumann boundary states with electric flux, as follows from some non-trivial $\vartheta$-function identity which we derive from first principles. Furthermore we discuss the GSO projection for the NS boundary states. In section [5] the analysis is repeated for the Ramond sector and in section [6] we fix the normalisation constants of the boundary states by comparison with the open string amplitude. Chapter 7 contains our conclusions. There are three appendices that describe some of the more technical calculations.

\section{The $N=2$ algebra and its free field realisation}

Let us begin by reviewing the $N=2$ superconformal algebra, and its realisation in terms of two free bosons and fermions.

The $N=2$ algebra consists of the energy momentum tensor, two supercharges $G^{ \pm}$and a $\mathrm{U}(1)$ current $J$. The commutation relations are given by [20]

$$
\begin{aligned}
{\left[L_{m}, L_{n}\right] } & =(m-n) L_{m+n}+\frac{c}{12}\left(m^{3}-m\right) \delta_{m,-n}, \\
{\left[L_{m}, J_{n}\right] } & =-n J_{m+n}, \\
{\left[L_{m}, G_{r}^{ \pm}\right] } & =\left(\frac{1}{2} m-r\right) G_{m+r}^{ \pm}, \\
{\left[J_{m}, J_{n}\right] } & =\frac{c}{3} m \delta_{m,-n}, \\
{\left[J_{m}, G_{r}^{ \pm}\right] } & = \pm G_{r+m}^{ \pm}, \\
\left\{G_{r}^{+}, G_{s}^{-}\right\} & =2 L_{r+s}+(r-s) J_{r+s}+\frac{c}{3}\left(r^{2}-\frac{1}{4}\right) \delta_{r,-s}, \\
\left\{G_{r}^{+}, G_{s}^{+}\right\} & =\left\{G_{r}^{-}, G_{s}^{-}\right\}=0,
\end{aligned}
$$

where $m, n$ are integers and $r, s$ are half-integers (integers) in the Neveu-Schwarz (Ramond) sector. For the free field realisation of the algebra in terms of two free bosons and fermions the central charge equals $c=3$. We will denote the eigenvalue of $L_{0}$ (the weight) by $h$ and the eigenvalue of $J_{0}$ (the charge) by $Q$.

In order to represent this algebra in terms of free fields we will arrange the two free bosonic fields $X^{1}(z, \bar{z}), X^{2}(z, \bar{z})$ and the two free real fermions $\psi^{1}(z), \psi^{2}(z)$ in complex pairs

$$
\begin{aligned}
Z & =\frac{1}{\sqrt{2}}\left(X^{1}+i X^{2}\right), & \bar{Z} & =\frac{1}{\sqrt{2}}\left(X^{1}-i X^{2}\right), \\
\psi^{+} & =\frac{1}{\sqrt{2}}\left(\psi^{1}+i \psi^{2}\right), & \psi^{-} & =\frac{1}{\sqrt{2}}\left(\psi^{1}-i \psi^{2}\right)
\end{aligned}
$$

with mode expansions (for the left-movers)

$$
\begin{array}{rlrl}
\partial Z=-i \sum_{m=-\infty}^{\infty} \alpha_{m} z^{-m-1}, & \partial \bar{Z} & =-i \sum_{m=-\infty}^{\infty} \bar{\alpha}_{m} z^{-m-1}, \\
\psi^{+}=\sum_{r} \psi_{r}^{+} z^{-r-1 / 2}, & \psi^{-}=\sum_{r} \psi_{r}^{-} z^{-r-1 / 2}
\end{array}
$$


These modes then have the commutation relations

$$
\begin{aligned}
{\left[\alpha_{m}, \bar{\alpha}_{n}\right] } & =m \delta_{m,-n}, \\
{\left[\alpha_{m}, \alpha_{n}\right] } & =\left[\bar{\alpha}_{m}, \bar{\alpha}_{n}\right]=0, \\
\left\{\psi_{r}^{+}, \psi_{s}^{-}\right\} & =\delta_{r,-s}, \\
\left\{\psi_{r}^{+}, \psi_{s}^{+}\right\} & =\left\{\psi_{r}^{-}, \psi_{s}^{-}\right\}=0 .
\end{aligned}
$$

Obviously, similar commutation relations hold for the corresponding right-moving degrees of freedom (that we shall denote by tildes in the following).

In terms of the free fields the left-moving $N=2$ algebra is given by

$$
\begin{aligned}
T & =-\partial Z \partial \bar{Z}-\frac{1}{2}\left(\psi^{-} \partial \psi^{+}+\psi^{+} \partial \psi^{-}\right), \\
G^{+} & =i \sqrt{2} \psi^{+} \partial \bar{Z} \\
G^{-} & =i \sqrt{2} \psi^{-} \partial Z \\
J & =-\psi^{-} \psi^{+},
\end{aligned}
$$

and we thus have the mode expansion for the left-moving $N=2$ generators

$$
\begin{aligned}
L_{n} & =\sum_{m}: \alpha_{n-m} \bar{\alpha}_{m}:+\frac{1}{2} \sum_{s}(2 s-n): \psi_{n-s}^{-} \psi_{s}^{+}:, \\
J_{n} & =-\sum_{s}: \psi_{-s}^{-} \psi_{s+n}^{+}: \\
G_{r}^{+} & =\sqrt{2} \sum_{m}: \bar{\alpha}_{m} \psi_{r-m}^{+}: \\
G_{r}^{-} & =\sqrt{2} \sum_{m}: \alpha_{m} \psi_{r-m}^{-}: .
\end{aligned}
$$

Obviously, the formulae for the right-moving generators are identical.

The irreducible representations of the free boson and fermion theory are labelled by the momenta of the ground state, $\left(\mathbf{p}_{L}, \mathbf{p}_{R}\right)$, where $\mathbf{p}_{L}$ and $\mathbf{p}_{R}$ are two-dimensional vectors. In general the momenta $\left(\mathbf{p}_{L}, \mathbf{p}_{R}\right)$ lie on a lattice (that characterises the structure of the twodimensional torus on which the theory is compactified); the results depend then in a somewhat complicated manner on the precise nature of this lattice. In order to avoid this difficulty we shall consider here the case where the theory is uncompactified. Then $\mathbf{p}:=\mathbf{p}_{L}=\mathbf{p}_{R}$, and $\mathbf{p}$ can take any value. The conformal weight of the momentum ground state labelled by $\mathbf{p}$ is $h=\tilde{h}=\frac{1}{2}\left(p_{1}^{2}+p_{2}^{2}\right)$, where $\mathbf{p}=\left(p_{1}, p_{2}\right)$.

The first step in constructing the most general $N=2$ boundary states for this theory consists of determining all $N=2$ Ishibashi states. To this end, we need to decompose the left- and right-moving representations of the free bosons and fermions in terms of $N=2$ representations. In the uncompactified theory, the left- and right-moving representations are obviously isomorphic, and it is therefore sufficient to concentrate just on the left-movers, say. We shall consider the NS and the R sector separately.

\subsection{The NS sector}

In the NS sector, it follows from (2.4) that each momentum ground state has $\mathrm{U}(1)$ charge $Q=0$. All $N=2$ highest weight representations for which the highest weight state has $Q=0$ 
are irreducible, unless $h=0$, i.e. unless the representation is the vacuum representation [21]. Thus for each $\mathbf{p} \neq 0$, the corresponding irreducible free field representation actually defines an irreducible representation of the $N=2$ algebra.

The situation is more interesting for the vacuum representation since the free field representation is then reducible with respect to the action of the $N=2$ algebra. In fact, the free field representation contains the $N=2$ singular vectors

$$
\begin{aligned}
& v_{(n)}^{(+)}=\left(\alpha_{-1}\right)^{n} \psi_{-1 / 2}^{+}|0\rangle, \\
& v_{(n)}^{(-)}=\left(\bar{\alpha}_{-1}\right)^{n} \psi_{-1 / 2}^{-}|0\rangle .
\end{aligned}
$$

It is shown in appendix $\mathrm{A}$ that these vectors are actual singular vectors with respect to the $N=2$ algebra. In fact, these are the only singular $N=2$ vectors, and we therefore have the decomposition

$$
\mathcal{H}_{0}^{\mathrm{NS}, \text { free }}=\mathcal{H}_{(0,0)}^{\mathrm{NS}, N=2} \bigoplus_{n=0}^{\infty}\left(\mathcal{H}_{\left(h=n+\frac{1}{2}, Q=1\right)}^{\mathrm{NS}, N=2} \oplus \mathcal{H}_{\left(h=n+\frac{1}{2}, Q=-1\right)}^{\mathrm{NS}, N=2}\right)
$$

where $\mathcal{H}_{(h, Q)}^{\mathrm{NS}, N=2}$ is the irreducible $N=2$ NS-representation with highest weight $h$ and charge $Q$. In order to see this, one observes that the character of the free field representation for any $\mathbf{p}$ is given by

$$
\chi_{\mathbf{p}}^{\mathrm{NS}, \text { free }}(q)=\operatorname{Tr} q^{2\left(L_{0}-\frac{c}{24}\right)}=q^{\mathbf{p}^{2}-\frac{1}{4}} \prod_{n=1}^{\infty} \frac{\left(1+q^{2 n-1}\right)^{2}}{\left(1-q^{2 n}\right)^{2}},
$$

where $q=e^{i \pi \tau}$. On the other hand, the characters of the $N=2, c=3$ irreducible highest weight representations are [22]

$$
\begin{gathered}
\chi_{(0,0)}^{\mathrm{NS}, N=2}(q)=\phi_{A}(q)\left(1-\frac{2 q}{1+q}\right) \\
\chi_{\left(h=\frac{2 n+1}{2}, Q= \pm 1\right)}^{\mathrm{NS}, N=2}(q)=q^{2 h} \phi_{A}(q)\left(1-q^{2}-\frac{q^{2 h}}{1+q^{2 h}}+\frac{q^{2 h+4}}{1+q^{2 h+2}}\right),
\end{gathered}
$$

where $\phi_{A}$ is defined as

$$
\phi_{A}(q):=q^{-\frac{1}{4}} \prod_{n=1}^{\infty} \frac{\left(1+q^{2 n-1}\right)^{2}}{\left(1-q^{2 n}\right)^{2}}
$$

It is thus easy to see that

$$
\chi_{0}^{\mathrm{NS}, \text { free }}(q)=\chi_{(0,0)}^{\mathrm{NS}, N=2}(q)+\sum_{n=0}^{\infty}\left[\chi_{\left(\frac{2 n+1}{2}, 1\right)}^{\mathrm{NS}, N=2}(q)+\chi_{\left(\frac{2 n+1}{2},-1\right)}^{\mathrm{NS}, N=2}(q)\right],
$$

which therefore implies that the free field representation cannot contain any further $N=2$ representations.

\section{$2.2 \quad$ The $\mathrm{R}$ sector}

In the Ramond sector the situation is similar. Any free field Ramond representation based on $\mathbf{p} \neq 0$ defines an irreducible $N=2$ representation. On the other hand, the vacuum 
representation is decomposable with respect to the $N=2$ algebra. The actual decomposition can be obtained from the result in the NS sector using the spectral flow $[22,23]$

$$
\mathcal{H}_{0}^{\mathrm{R}, \text { free }}=\mathcal{H}_{\left(\frac{1}{8}, \frac{1}{2}\right)}^{\mathrm{R}, N=2} \oplus \mathcal{H}_{\left(\frac{1}{8},-\frac{1}{2}\right)}^{\mathrm{R}, N=2} \bigoplus_{n=1}^{\infty}\left(\mathcal{H}_{\left(n+\frac{1}{8}, \frac{3}{2}\right)}^{\mathrm{R}, N=2} \oplus \mathcal{H}_{\left(n+\frac{1}{8},-\frac{3}{2}\right)}^{\mathrm{R}, N=2}\right),
$$

where $\mathcal{H}_{(h, Q)}^{\mathrm{R}, N=2}$ is the irreducible $N=2$ R-representation with highest weight $h$ and charge $Q$. We should note that the two Ramond ground states $\left|\frac{1}{8}, \pm \frac{1}{2}\right\rangle$ are not related to one another by the $N=2$ algebra,

$$
G_{0}^{ \pm}\left|\frac{1}{8}, \frac{1}{2}\right\rangle=G_{0}^{ \pm}\left|\frac{1}{8},-\frac{1}{2}\right\rangle=0,
$$

but appear both in the free field representation since

$$
\psi_{0}^{\mp}\left|\frac{1}{8}, \pm \frac{1}{2}\right\rangle=\left|\frac{1}{8}, \mp \frac{1}{2}\right\rangle .
$$

They give therefore rise to two separate $N=2$ representations. The other $N=2$ representations that appear are generated by the singular vectors

$$
\begin{aligned}
& w_{(n)}^{(+,+)}=\left(\alpha_{-1}\right)^{n-1} \psi_{-1}^{+}\left|\frac{1}{8}, \frac{1}{2}\right\rangle, \\
& w_{(n)}^{(+, 0)}=\left(\alpha_{-1}\right)^{n}\left|\frac{1}{8}, \frac{1}{2}\right\rangle=G_{0}^{-} w_{(n)}^{(+,+)}
\end{aligned}
$$

and

$$
\begin{aligned}
& w_{(n)}^{(-,-)}=\left(\bar{\alpha}_{-1}\right)^{n-1} \psi_{-1}^{-}\left|\frac{1}{8},-\frac{1}{2}\right\rangle, \\
& w_{(n)}^{(-, 0)}=\left(\bar{\alpha}_{-1}\right)^{n}\left|\frac{1}{8},-\frac{1}{2}\right\rangle=G_{0}^{+} w_{(n)}^{(-,-)},
\end{aligned}
$$

where $n \in \mathbb{N}$. The first two singular vectors $w_{(n)}^{(+,+)}$and $w_{(n)}^{(+, 0)}$ (that are related to one another by the action of $\left.G_{0}^{ \pm}\right)$generate the R-representation $\mathcal{H}_{\left(n+\frac{1}{8}, \frac{3}{2}\right)}^{\mathrm{R}, N=2}$, while the two singular vectors $w_{(n)}^{(-,-)}$and $w_{(n)}^{(-, 0)}$ generate $\mathcal{H}_{\left(n+\frac{1}{8},-\frac{3}{2}\right)}^{\mathrm{R}, N=2}$.

Again (2.11) is consistent with the corresponding character formulae. For the free field representation we simply have

$$
\chi_{0}^{\mathrm{R}, \text { free }}(q)=2 \prod_{n=1}^{\infty} \frac{\left(1+q^{2 n}\right)^{2}}{\left(1-q^{2 n}\right)^{2}},
$$

while the $N=2$ characters are

$$
\begin{aligned}
\chi_{\left(\frac{1}{8}, \pm \frac{1}{2}\right)}^{\mathrm{R}, N=2}(q) & =\phi_{P}(q)\left(1-\frac{q^{2}}{1+q^{2}}-\frac{1}{2}\right) \\
\chi_{\left(n+\frac{1}{8}, \pm \frac{3}{2}\right)}^{\mathrm{R}, N=2}(q) & =q^{2 n} \phi_{P}(q)\left(1-q^{2}-\frac{q^{2 n}}{1+q^{2 n}}+\frac{q^{2 n+4}}{1+q^{2 n+2}}\right)
\end{aligned}
$$

with

$$
\phi_{P}(q):=2 \prod_{n=1}^{\infty} \frac{\left(1+q^{2 n}\right)^{2}}{\left(1-q^{2 n}\right)^{2}}
$$


As in the NS case it is then obvious that

$$
\chi_{0}^{\mathrm{R}, \text { free }}(q)=\chi_{\left(\frac{1}{8}, \frac{1}{2}\right)}^{\mathrm{R}, N=2}(q)+\chi_{\left(\frac{1}{8}, \frac{-1}{2}\right)}^{\mathrm{R}, N=2}(q)+\sum_{n=1}^{\infty}\left(\chi_{\left(n+\frac{1}{8}, \frac{3}{2}\right)}^{\mathrm{R}, N=2}(q)+\chi_{\left(n+\frac{1}{8},-\frac{3}{2}\right)}^{\mathrm{R}, N=2}(q)\right) .
$$

Note that in order to distinguish representations that only differ by their U(1) charge it would be necessary to include in the trace the operator $z^{J_{0}}$; however, the specialised characters used above are already sufficient for the construction of the boundary states that shall be considered in the following.

\section{Construction of the boundary states}

Now we want to construct the boundary states which preserve the $N=2$ superconformal algebra. There are two possible gluing conditions which can be imposed on the boundary. They are usually referred to as A-type and B-type boundary conditions [8]. In terms of boundary conformal field theory, B-type boundary conditions correspond to the identity gluing automorphism, whereas A-type boundary conditions involve the mirror automorphism [24]. More specifically, the A-type gluing conditions read

$$
\begin{aligned}
\left.\left.\left(L_{n}-\tilde{L}_{-n}\right) \| B\right\rangle\right\rangle_{A} & =0, \\
\left.\left.\left(J_{n}-\tilde{J}_{-n}\right) \| B\right\rangle\right\rangle_{A} & =0, \\
\left.\left.\left(G_{r}^{+}+i \eta \tilde{G}_{-r}^{-}\right) \| B\right\rangle\right\rangle_{A} & =0, \\
\left.\left.\left(G_{r}^{-}+i \eta \tilde{G}_{-r}^{+}\right) \| B\right\rangle\right\rangle_{A} & =0,
\end{aligned}
$$

while the B-type gluing conditions are

$$
\begin{aligned}
\left.\left.\left(L_{n}-\tilde{L}_{-n}\right) \| B\right\rangle\right\rangle_{B} & =0, \\
\left.\left.\left(J_{n}+\tilde{J}_{-n}\right) \| B\right\rangle\right\rangle_{B} & =0, \\
\left.\left.\left(G_{r}^{+}+i \eta \tilde{G}_{-r}^{+}\right) \| B\right\rangle\right\rangle_{B} & =0, \\
\left.\left.\left(G_{r}^{-}+i \eta \tilde{G}_{-r}^{-}\right) \| B\right\rangle\right\rangle_{B} & =0 .
\end{aligned}
$$

Here $\eta= \pm$ describes the different spin structures. For the free field situation considered here, A-type and B-type gluing conditions are essentially equivalent (since 'mirror symmetry' corresponds simply to T-duality in one direction); we shall therefore concentrate on the technically simpler B-type case in the following.

These gluing conditions have only non-trivial solutions for the NS-NS and the R-R sector. In this section we want to consider the component of the boundary state in the NS-NS sector; the construction for the R-R sector (that is fairly similar) will be done in section 5 .

\subsection{The boundary states in the NS-NS sector}

In the first step we need to identify all the $N=2$ Ishibashi states that arise in the NSNS sector. Given that the left- and right-moving representations are isomorphic (as free field representations), and that we consider the trivial gluing condition, it follows from the standard arguments that there is precisely one $N=2$ Ishibashi state for each irreducible $N=2$ representation that appears in the above decompositions [25]. In particular, we therefore 
have one $N=2$ Ishibashi state for each $\mathbf{p} \neq 0$; this Ishibashi state must therefore agree with the usual U(1) Dirichlet Ishibashi state. By considering the usual Dirichlet branes, we can construct boundary states that couple to all of these Ishibashi states, and they therefore do not give rise to any new $N=2$ boundary states.

The situation is more interesting for the vacuum sector. For the $\mathrm{U}(1)$ theory, there is one Ishibashi state (for each gluing condition) that can be associated to this sector; more specifically, we have one U(1) Dirichlet and one U(1) Neumann Ishibashi state (for each choice of $\eta$ ) from the vacuum sector. However, in terms of the $N=2$ algebra, there are infinitely many different Ishibashi states. In fact, because of (2.6) we have an $N=2$ Ishibashi state for each $(n, Q)$, where $(n, Q)=(0,0)$ or $n \in \mathbb{N}$ and $Q= \pm 1$. In order to introduce a uniform notation, we denote these $N=2$ Ishibashi states as

$$
|n, \eta\rangle\rangle= \begin{cases}|(n, 1), \eta\rangle\rangle & \text { for } n>0 \\ |(0,0), \eta\rangle\rangle & \text { for } n=0 \\ |(|n|,-1), \eta\rangle\rangle & \text { for } n<0\end{cases}
$$

We fix the normalisation of these Ishibashi states by demanding that the usual Neumann $\mathrm{U}(1)$ boundary state

$$
\| N, \eta\rangle\rangle^{\text {free }}=\mathcal{N} \exp \left[-\sum_{n>0} \frac{1}{n}\left(\alpha_{-n} \tilde{\bar{\alpha}}_{-n}+\bar{\alpha}_{-n} \tilde{\alpha}_{-n}\right)-i \eta \sum_{r>0}\left(\psi_{-r}^{+} \tilde{\psi}_{-r}^{-}+\psi_{-r}^{+} \tilde{\psi}_{-r}^{-}\right)\right]|0\rangle,
$$

where $\mathcal{N}$ is a normalisation constant, equals

$$
\left.\| N, \eta\rangle\rangle^{\text {free }}=\mathcal{N} \sum_{n \in \mathbb{Z}}|n, \eta\rangle\right\rangle
$$

Clearly, this is not the most general $N=2$ boundary state since the coefficients in front of the different $N=2$ Ishibashi states do not all have to be equal. The most general ansatz for a $N=2$ boundary state from the NS-NS sector is

$$
\left.\| C, \eta\rangle\rangle^{N=2}=\sum_{n \in \mathbb{Z}} C_{n}|n, \eta\rangle\right\rangle
$$

where $C_{n}$ are constants that need to be determined, using the usual consistency conditions, in particular Cardy's condition [26].

Given that the $N=2$ Ishibashi states are labelled by the integers, it is very suggestive that (up to an overall normalisation), the $C_{n}$ should simply equal

$$
C_{n}=e^{i n \phi}
$$

where $\phi$ is an angular variable. Thus our ansatz for the boundary state is

$$
\left.\| N, \phi, \eta\rangle\rangle=\mathcal{N}_{N S}(\phi) \sum_{n \in \mathbb{Z}} e^{i n \phi}|n, \eta\rangle\right\rangle
$$

where $\mathcal{N}_{N S}(\phi)$ is a normalisation constant that will be determined below. It is clear that if this family of boundary states is in fact consistent for all $\phi$, then it is already the most general class of boundary states. This is simply a consequence of the fact that we can invert 
(essentially by Fourier transformation) the above relation to write every Ishibashi state $|n, \eta\rangle\rangle$ as an integral over the boundary states $\| N, \phi, \eta\rangle\rangle$. Any boundary state of the form (3.6) can therefore be expressed as a linear combination (or possibly an integral) of these boundary states. In this sense the above boundary states are therefore generating all boundary states (if they are indeed consistent).

On the other hand, given the structure of the singular vectors (2.5), comparison of (3.5) with (3.4) suggests that this ansatz simply corresponds to switching on an electric field on the usual Neumann brane; in terms of the gluing conditions of the free fields this means that

$$
\begin{aligned}
\left.\left.\left(\alpha_{n}+e^{-i \phi} \tilde{\alpha}_{-n}\right) \| N, \phi, \eta\right\rangle\right\rangle^{\text {free }} & =0, \\
\left.\left.\left(\bar{\alpha}_{n}+e^{i \phi} \tilde{\bar{\alpha}}_{-n}\right) \| N, \phi, \eta\right\rangle\right\rangle^{\text {free }} & =0, \\
\left.\left.\left(\psi_{r}^{+}+i \eta e^{-i \phi} \tilde{\psi}_{-r}^{+}\right) \| N, \phi, \eta\right\rangle\right\rangle^{\text {free }} & =0, \\
\left.\left.\left(\psi_{r}^{-}+i \eta e^{i \phi} \tilde{\psi}_{-r}^{-}\right) \| N, \phi, \eta\right\rangle\right\rangle^{\text {free }} & =0 .
\end{aligned}
$$

It is clear from (2.4) that this automorphism acts trivially on the $N=2$ generators, and therefore that it leaves the $N=2$ gluing conditions unmodified.

In the following section we shall confirm that this expectation is indeed correct; more specifically, we shall show that the cylinder amplitudes of (3.8) agree with those of a Neumann brane with an electric field. This will then also allow us to determine the correct normalisation constants $\mathcal{N}_{N S}(\phi)$.

\section{Calculation of the amplitude}

In this section we want to calculate the overlap of two NS-NS boundary states of the form (3.8). Recall that our normalisation convention for the $N=2$ Ishibashi states implies that

$$
\left\langle\left\langle n\left|q^{L_{0}+\tilde{L}_{0}-\frac{1}{4}}\right| n^{\prime}\right\rangle\right\rangle=\chi_{n}(q) \delta_{n, n^{\prime}},
$$

where $\chi_{n}$ denotes the appropriate $N=2$ character. We shall first consider the situation where the two spin structures are the same (but the phases $\phi$ may differ)

$$
\begin{aligned}
\mathcal{A}_{\phi_{1}, \phi_{2}}^{+} & =\left\langle\left\langle N, \phi_{1}, \eta\left\|q^{L_{0}+\tilde{L}_{0}-\frac{1}{4}}\right\| N, \phi_{2}, \eta\right\rangle\right\rangle \\
& =\mathcal{N}_{N S}\left(\phi_{1}\right) \mathcal{N}_{N S}\left(\phi_{1}\right) \sum_{n \in \mathbb{Z}} e^{i n\left(\phi_{1}-\phi_{2}\right)} \chi_{n}(q) .
\end{aligned}
$$

If we set $\Delta:=\phi_{1}-\phi_{2}$ we can rewrite the sum in (4.2) as

$$
\begin{aligned}
\sum_{n \in \mathbb{Z}} e^{i n \Delta} \chi_{n}(q) & =\chi_{0}(q)+\sum_{n>0}\left(e^{i n \Delta}+e^{-i n \Delta}\right) \chi_{n}(q) \\
= & q^{-\frac{1}{4}} \prod_{n=1}^{\infty} \frac{\left(1+q^{2 n-1}\right)^{2}}{\left(1-q^{2 n}\right)^{2}}\left[1+2 \sum_{n=1}^{\infty}[\cos (n \Delta)-\cos ((n-1) \Delta)] \frac{q^{2 n-1}}{1+q^{2 n-1}}\right] .
\end{aligned}
$$

As was explained in the previous section, we expect this amplitude to agree with that of D-branes with electric flux (labelled by $\phi_{1}$ and $\phi_{2}$, respectively). The cylinder amplitude for two such branes is given by (see for example $[27,28]$ )

$$
\mathcal{A}^{\text {electric }}=\left(\cos \frac{\phi_{1}}{2} \cos \frac{\phi_{2}}{2}\right)^{-1} q^{-\frac{1}{4}} \frac{\prod_{n=1}^{\infty}\left(1+2 \cos (\Delta) q^{2 n-1}+q^{4 n-2}\right)}{\prod_{n=1}^{\infty}\left(1-2 \cos (\Delta) q^{2 n}+q^{4 n}\right)} .
$$


In terms of $\vartheta$-functions formula (4.4) can be written as

$$
\mathcal{A}^{\text {electric }}=\frac{2 \sin \left(\frac{\Delta}{2}\right)}{\cos \frac{\phi_{1}}{2} \cos \frac{\phi_{2}}{2}} \frac{\vartheta_{3}\left(\frac{\Delta}{2}, q\right)}{\vartheta_{1}\left(\frac{\Delta}{2}, q\right)},
$$

which makes its modular properties manifest. (The relevant $\vartheta$-functions are defined in appendix $B$ where their modular properties are also reviewed.) It is a non-trivial fact that these two expressions, i.e. (4.3) and (4.4), are actually equal (up to normalisation); this is proven in appendix [C] With the help of this result, and keeping track of the various normalisation constants, we then find that

$$
\mathcal{A}_{\phi_{1}, \phi_{2}}^{+}=2 \mathcal{N}_{N S}\left(\phi_{1}\right) \mathcal{N}_{N S}\left(\phi_{2}\right) \sin \left(\frac{\Delta}{2}\right) \frac{\vartheta_{3}\left(\frac{\Delta}{2}, q\right)}{\vartheta_{1}\left(\frac{\Delta}{2}, q\right)},
$$

where the $\mathcal{N}_{N S}\left(\phi_{i}\right)$ are the same constants that appeared in (3.8).

\subsection{GSO projection}

Up to now we have only considered one spin structure, but in order to obtain spacetime supersymmetric branes, we need to impose a GSO-projection, and this will require that we consider both spin structures together. The GSO-invariant NS-NS boundary states can be constructed by the following standard method (see for example [32]). Strictly speaking, when considering the GSO-projection we have to specify the superconformal field theory that makes up the remaining central charge and apply the GSO-projection to the full theory. In order to avoid having to make a choice for this additional theory we restrict ourselves in the following to GSO-projecting the boundary states of our $c=3$ theory separately; the modifications that arise when including the remaining degrees of freedom in the full theory are straightforward. Let us assume that the relevant projection for the $c=3$ theory in the NS-NS sector is simply

$$
P_{G S O}=\frac{1}{4}\left(1+(-1)^{F}\right)\left(1+(-1)^{\tilde{F}}\right) .
$$

By definition, the action of the world-sheet fermion number operator on the NS-NS ground state is given by

$$
(-1)^{\tilde{F}}|0,0\rangle=(-1)^{F}|0,0\rangle=-|0,0\rangle .
$$

$(-1)^{F}$ anticommutes with fermionic modes, and its action on the singular vectors given by (2.5) is therefore

$$
(-1)^{F} v_{(n)}^{( \pm)}=+v_{(n)}^{( \pm)}
$$

The GSO-invariant combinations of Ishibashi states are therefore

$$
|n\rangle\rangle= \begin{cases}\left.\left.\frac{1}{\sqrt{2}}(|n, \eta\rangle\rangle+|n,-\eta\rangle\right\rangle\right), & \text { for } n \neq 0 \\ \left.\left.\frac{1}{\sqrt{2}}(|0, \eta\rangle\rangle-|0,-\eta\rangle\right\rangle\right), & \text { for } n=0 .\end{cases}
$$

Our ansatz for the total boundary state is thus

$$
\left.\| N, \phi\rangle=\mathcal{N}_{N S}(\phi) \sum_{n \in \mathbb{Z}} e^{i n \phi}|n\rangle\right\rangle,
$$

where $\mathcal{N}_{N S}(\phi)$ is a normalisation constant that will be determined below. 
The cylinder diagram of two such GSO-invariant boundary states is now

$$
\left\langle\left\langle N, \phi_{1}\left\|q^{\left(L_{0}+\tilde{L}_{0}-\frac{1}{4}\right)}\right\| N, \phi_{2}\right\rangle\right\rangle=\left(\mathcal{A}_{\phi_{1}, \phi_{2}}^{+}+\mathcal{A}_{\phi_{1}, \phi_{2}}^{-}\right)
$$

where $\mathcal{A}_{\phi_{1}, \phi_{2}}^{+}$is given in (4.6), while $\mathcal{A}_{\phi_{1}, \phi_{2}}^{-}$is the contribution from $\eta_{1}=-\eta_{2}$. Because of the sign structure of (4.9) this equals

$$
\begin{aligned}
\mathcal{A}_{\phi_{1}, \phi_{2}}^{-} & =\mathcal{N}_{N S}\left(\phi_{1}\right) \mathcal{N}_{N S}\left(\phi_{2}\right) \times \\
& \times q^{-1 / 4} \prod_{n=1}^{\infty} \frac{\left(1-q^{2 n-1}\right)^{2}}{\left(1-q^{2 n}\right)^{2}}\left[-1-\frac{2 q}{1-q}+2 \sum_{n} \cos (n \Delta)\left(\frac{q^{2 n-1}}{1-q^{2 n-1}}-\frac{q^{2 n+1}}{1-q^{2 n+1}}\right)\right] .
\end{aligned}
$$

This can be expressed, in terms of $\vartheta$-functions, as

$$
\mathcal{A}_{\phi_{1}, \phi_{2}}^{-}=-2 \mathcal{N}_{N S}\left(\phi_{1}\right) \mathcal{N}_{N S}\left(\phi_{2}\right) \sin \left(\frac{\Delta}{2}\right) \frac{\vartheta_{4}\left(\frac{\Delta}{2}, q\right)}{\vartheta_{1}\left(\frac{\Delta}{2}, q\right)} .
$$

This follows by a similar identity as the one used before, and can be proven by the method of appendix C

\section{The Ramond Sector}

Next we want to perform the analogous analysis in the R-R sector. Most of the analysis will be the same as for the NS-NS sector.

First of all, if $\mathbf{p} \neq 0$, there is precisely one $N=2$ Ishibashi state (for each choice of $\eta$ ), which therefore agrees with the usual Dirichlet Ishibashi state. Again these Ishibashi states appear in the boundary states for the usual Dirichlet branes, and do not give rise to any interesting new D-branes.

As in the NS-NS sector, the situation is more interesting in the vacuum sector. By the usual argument we get one Ishibashi state for each irreducible representation that appears in (2.11). It is convenient to label the Ishibashi states by $|n, \pm, \eta\rangle\rangle$ for $n>0$, as well as $|0,+, \eta\rangle\rangle$ and $|0,-, \eta\rangle\rangle$. (Thus for example the Ishibashi state $|n, \pm, \eta\rangle\rangle$ has the expansion

$$
\begin{aligned}
|n,+, \eta\rangle\rangle & \sim w_{(n)}^{(+,+)} \otimes \tilde{w}_{(n)}^{(-,-)}+\cdots \\
|n,-, \eta\rangle\rangle & \left.\sim w_{(n)}^{(-,-)} \otimes \tilde{w}_{(n)}^{(+,+)}+\cdots .\right)
\end{aligned}
$$

Furthermore, as in the NS-NS sector, we fix its normalisation by demanding that the usual Neumann boundary state is simply given as

$$
\left.\left.\left.\left.\| N, \phi, \eta\rangle\rangle^{\text {free }}=\mathcal{N}[|0,+, \eta\rangle\rangle+|0,-, \eta\rangle\right\rangle+\sum_{n \in \mathbb{N}}(|n,+, \eta\rangle\rangle+|n,-, \eta\rangle\right\rangle\right)\right],
$$

where $\mathcal{N}$ is some normalisation constant. As before in the NS-NS sector, this is not the most general $N=2$ boundary state; in order to obtain a spacetime supersymmetric brane, we need to mimic the construction in the NS-NS sector, and we therefore expect that we should 
simply switch on an electric field on the world-volume of the brane. Given the structure of the $N=2$ singular vectors in terms of the free fields, this suggests the ansatz

$$
\begin{aligned}
&\| R, \phi, \eta\rangle\rangle=\left.\mathcal{N}_{R}(\phi)\left(e^{\frac{i}{2} \phi}|0,+, \eta\rangle\right\rangle+e^{-\frac{i}{2} \phi}|0,-, \eta\rangle\right\rangle+ \\
&\left.\left.\left.\sum_{n \in \mathbb{N}} e^{i\left(n+\frac{1}{2}\right) \phi}|n,+, \eta\rangle\right\rangle+e^{-i\left(n+\frac{1}{2}\right) \phi}|n,-, \eta\rangle\right\rangle\right) .
\end{aligned}
$$

By the same reasoning as before in the NS-NS sector, these boundary states will account for all Ishibashi states (if they are indeed consistent) since we can invert this relation and write the Ishibashi states in terms of these boundary states.

The overlap between two such boundary states now becomes

$$
\begin{aligned}
\mathcal{A}_{\phi_{1}, \phi_{2}}^{R} & =\left\langle\left\langle R, \phi_{1}, \eta\left\|q^{L_{0}+\tilde{L}_{0}+\frac{1}{8}}\right\| R, \phi_{2}, \eta\right\rangle\right\rangle \\
& =\mathcal{N}_{R}\left(\phi_{1}\right) \mathcal{N}_{R}\left(\phi_{2}\right)\left(2 \cos (\Delta / 2) \chi_{0}^{\mathrm{R}}(q)+2 \sum_{n=1}^{\infty} \cos ((n+1 / 2) \Delta) \chi_{n}^{\mathrm{R}}(q)\right),
\end{aligned}
$$

where $\Delta=\phi_{1}-\phi_{2}$ as before, and $\chi_{n}^{\mathrm{R}}(q)$ denotes the character (2.17). Again, we can express the amplitude in terms of $\vartheta$-functions (using a relation similar to the one proven in appendix C), and we thus obtain

$$
\mathcal{A}_{\phi_{1}, \phi_{2}}^{R}=2 \mathcal{N}_{R}\left(\phi_{1}\right) \mathcal{N}_{R}\left(\phi_{2}\right) \sin \left(\frac{\Delta}{2}\right) \frac{\vartheta_{2}\left(\frac{\Delta}{2}, q\right)}{\vartheta_{1}\left(\frac{\Delta}{2}, q\right)} .
$$

\subsection{GSO projection}

As before, we need to make sure that the boundary state is invariant under the GSOprojection. In the presence of fermionic zero modes, this requires (as always) a little bit of care. We choose the convention that

$$
(-1)^{F}\left|\frac{1}{8}, \frac{1}{2}\right\rangle=+\left|\frac{1}{8}, \frac{1}{2}\right\rangle, \quad(-1)^{\tilde{F}}\left|\frac{1}{8}, \frac{1}{2}\right\rangle=+\left|\frac{1}{8}, \frac{1}{2}\right\rangle .
$$

It is always possible to choose the normalisation of the Ishibashi states so that

$$
\left.\left.(-1)^{F}|n, Q, \eta\rangle\right\rangle=|n, Q,-\eta\rangle\right\rangle,
$$

but then the action of $(-1)^{\tilde{F}}$ is unambiguous. Given the form of the Ishibashi states (5.1a), (5.1b), as well as our conventions about the action of the fermion number operators (5.6) it is then easy to see that

$$
\left.\left.(-1)^{\tilde{F}}|n, Q, \eta\rangle\right\rangle=-|n, Q,-\eta\rangle\right\rangle \text {. }
$$

(The same also holds for the two Ishibashi states that come from the $\mathrm{R}$ ground states.) In order to obtain consistent boundary states we have to chose the GSO projector in the R sector as a type IIA GSO projector ${ }^{1}$

$$
P_{G S O}=\frac{1}{4}\left(1+(-1)^{F}\right)\left(1-(-1)^{\tilde{F}}\right)
$$

\footnotetext{
${ }^{1}$ This does not mean that these boundary states only exist in the complete ten-dimensional theory if it is type IIA; however it does give constraints on whether this Neumann boundary condition for the $c=3$ sector is consistent within the context of the complete theory. The fact that a IIA projection appears simply reflects the fact that we are describing a D2-brane here.
} 
and the GSO invariant states are then given by

$$
\left.\left.|n, \pm\rangle\rangle_{R R}=\frac{1}{\sqrt{2}}(|n, \pm,+\rangle\rangle-|n, \pm,-\rangle\right\rangle\right)
$$

with a similar formula for $|0, \pm\rangle\rangle$. The GSO-invariant ansatz for our boundary state is then simply

$$
\left.\left.\left.\left.\| R, \phi\rangle\rangle=\mathcal{N}_{R}(\phi)\left(e^{\frac{i}{2} \phi}|0,+\rangle\right\rangle+e^{-\frac{i}{2} \phi}|0,-\rangle\right\rangle+\sum_{n \in \mathbb{N}} e^{i\left(n+\frac{1}{2}\right) \phi}|n,+\rangle\right\rangle+e^{-i\left(n+\frac{1}{2}\right) \phi}|n,-\rangle\right)\right) .
$$

The calculation of the cylinder amplitude involving these GSO-invariant boundary states is trivial since the overlap between two boundary states with opposite $\eta$ vanishes. Thus the formula simply reproduces again (5.5).

\section{The open string amplitude and normalisation}

Finally, in order to fix the normalisation constants and to check that our results are consistent with the open string results, we perform a modular transformation on the amplitudes to the open string sector. We make the ansatz for our boundary state to be simply

$$
\left.\left.\left.\left.\| \phi\rangle\rangle=\frac{1}{\sqrt{2}}(\| N, \phi\rangle\right\rangle+i \epsilon \| R, \phi\right\rangle\right)\right)
$$

where $\epsilon= \pm 1$ distinguishes a brane from an anti-brane. Using the results we have derived in the previous sections, it is easy to see that the cylinder amplitude between two such branes gives rise, in the open string description, to the amplitude

$$
\begin{aligned}
\mathcal{A}=i \mathcal{N}_{N S}\left(\phi_{1}\right) \mathcal{N}_{N S}\left(\phi_{2}\right) \sin \left(\frac{\Delta}{2}\right)\left[\frac{\vartheta_{3}\left(\frac{\Delta \tilde{\tau}}{2}, \tilde{q}\right)}{\vartheta_{1}\left(\frac{\Delta \tilde{\tau}}{2}, \tilde{q}\right)}-\frac{\vartheta_{2}\left(\frac{\Delta \tilde{\tau}}{2}, \tilde{q}\right)}{\vartheta_{1}\left(\frac{\Delta \tilde{\tau}}{2}, \tilde{q}\right)}\right] \\
-i \mathcal{N}_{R}\left(\phi_{1}\right) \mathcal{N}_{R}\left(\phi_{2}\right) \sin \left(\frac{\Delta}{2}\right) \frac{\vartheta_{4}\left(\frac{\Delta \tilde{\tau}}{2}, \tilde{q}\right)}{\vartheta_{1}\left(\frac{\Delta \tilde{\tau}}{2}, \tilde{q}\right)},
\end{aligned}
$$

where $\tilde{\tau}=-\frac{1}{\tau}$ and $\tilde{q}=e^{i \pi \tilde{\tau}}$. In deriving this formula we have used the modular transformation properties of the $\vartheta$-functions that are detailed in the appendix.

The above formula now suggests that we should set $\mathcal{N}_{N S}(\phi)=\mathcal{N}_{R}(\phi)$; furthermore, since these amplitudes are the same as for an open string in the presence of a background electric field, the analysis of [29] (see also $[27,30,31]$ ) suggests that

$$
\mathcal{N}_{N S}(\phi)=\mathcal{N}_{R}(\phi)=\frac{1}{\cos \frac{\phi}{2}}
$$

For example, the first term in (6.2a) then becomes after a short calculation

$$
\frac{1}{2}\left(\tan \left(\frac{\phi_{1}}{2}\right)-\tan \left(\frac{\phi_{2}}{2}\right)\right) \frac{\tilde{q}^{\frac{\Delta}{2 \pi}}}{1-\tilde{q}^{\frac{\Delta}{\pi}}} \tilde{q}^{-1 / 4} \prod_{n=1}^{\infty} \frac{\left(1+e^{i \Delta \tilde{\tau}} \tilde{q}^{2 n-1}\right)\left(1+e^{-i \Delta \tilde{\tau}} \tilde{q}^{2 n-1}\right)}{\left(1-e^{i \Delta \tilde{\tau}} \tilde{q}^{2 n}\right)\left(1-e^{-i \Delta \tilde{\tau}} \tilde{q}^{2 n}\right)} .
$$

This is the correct mode expansion for (one half of) the open string NS sector [27,30,31]. The other terms work similarly; in particular, the contribution from the RR boundary states gives rise to one half of the open string NS sector with the insertion of $(-1)^{F}$, and thus combines with (6.4) to give the correct GSO-projection. 


\section{Conclusion}

In this paper we have constructed the most general $N=2$ superconformal boundary states for the theory of two free (uncompactified) bosons and fermions. We have shown that the only $N=2$ D-branes for this theory are the usual Dirichlet branes, as well as the Neumann branes with an electric field. In the process of identifying the $N=2$ boundary states with Neumann branes with electric flux we were led to prove some non-trivial $\vartheta$-function identities that may be interesting in their own right.

Our analysis implies in particular that for this theory the usual Neumann and Dirichlet branes already account for all $N=2$ D-branes. This is in marked contrast to the results in the non-supersymmetric case, or the case with $N=1$ supersymmetry, where there exist conformal or $N=1$ superconformal D-branes that do not have an interpretation in terms of Neumann or Dirichlet branes [4].

On the other hand, in contradistinction to the cases mentioned above, the boundary states we have constructed in this paper preserve $N=2$ worldsheet supersymmetry, and the corresponding D-branes are therefore spacetime supersymmetric and stable. Our results therefore imply that the usual Neumann and Dirichlet branes already account for all spacetime supersymmetric D-branes in this simple example. This is in some sense reassuring since until now mostly these D-branes have been considered.

There are indications that there exist other theories (for example certain orbifold theories [33]) for which the supersymmetric D-branes charges are not just generated by the usual Neumann and Dirichlet branes. One should hope that the techniques of this paper will help to construct those branes.

\section{Acknowledgements}

We would like to thank Terry Gannon, Kevin Graham, Peter Kaste, Tako Mattik, Andreas Recknagel and Daniel Roggenkamp for interesting discussions. This research is partially supported by the Swiss National Science Foundation. The work of H.K. is also supported by a scholarship of the Marianne und Dr. Fritz Walter Fischer-Stiftung and a Promotionsstipendium of the Deutscher Akademischer Austauschdienst (DAAD).

\section{A Singular vectors}

In this appendix we want to show that the vectors given in equation (2.5), namely

$$
\begin{aligned}
& v_{(n)}^{(+)}=\left(\alpha_{-1}\right)^{n} \psi_{-1 / 2}^{+}|0\rangle \\
& v_{(n)}^{(-)}=\left(\bar{\alpha}_{-1}\right)^{n} \psi_{-1 / 2}^{-}|0\rangle
\end{aligned}
$$

are actually singular vectors for the $N=2$ algebra.

The mode expansions of the $N=2$ operators in terms of the free fields are given in equations (2.4). We need to prove that $S_{m} v=0$ for $m>0$, where $S$ stands for any generator of the $N=2$ algebra. Consider for example the situation where $S=L$,

$$
L_{m} v_{(n)}^{(+)}, \quad m>0
$$


which, in terms of the free field modes, is given by

$$
\left(\sum_{n}: \alpha_{m-n} \bar{\alpha}_{n}:+\frac{1}{2} \sum_{s}(2 s-m): \psi_{m-s}^{-} \psi_{s}^{+}:\right)\left(\alpha_{-1}\right)^{n} \psi_{-1 / 2}^{+}|0\rangle .
$$

Let us focus on the bosonic part first. Due to the (anti-)commutation relations given in (2.2), it is clear that all products in the expansion of $L_{m}$ which do not contain $\bar{\alpha}_{1}$ contain an operator with positive mode number which commutes with the creation operators that appear in the singular vector and annihilates the ground state. Therefore the only terms we have to consider more closely are of the form $\alpha_{m-1} \bar{\alpha}_{1}$. By assumption $m \geq 1$ and therefore $m-1 \geq 0$. Thus any term of this form contains an annihilation operator which again commutes with the operators in the singular vector, and thus annihilates the ground state. This shows that the bosonic part of $L_{m}$ annihilates $v_{n}$. The argument for the fermionic part runs along the same lines. The same argument works also for the other $N=2$ generators, except that for the supercharges one has to use additionally that $\left(\psi_{-1 / 2}^{+}\right)^{2}=0$.

\section{B Various $\vartheta$ function definitions and identities}

This is a list of $\vartheta$ function definitions and identities, collected at various points; in particular [34] and [35] are useful references.

Definition of $\vartheta$-functions

The $\vartheta$-functions are defined as

$$
\begin{aligned}
& \vartheta_{1}(z, q)=2 \sum_{n=0}^{\infty}(-1)^{n} q^{\left(n+\frac{1}{2}\right)^{2}} \sin (2 n+1) z \\
& \vartheta_{2}(z, q)=2 \sum_{n=0}^{\infty} q^{\left(n+\frac{1}{2}\right)^{2}} \cos (2 n+1) z \\
& \vartheta_{3}(z, q)=1+2 \sum_{n=1}^{\infty} q^{n^{2}} \cos 2 n z \\
& \vartheta_{4}(z, q)=1+2 \sum_{n=1}^{\infty}(-1)^{n} q^{n^{2}} \cos 2 n z
\end{aligned}
$$

where $q=e^{i \pi \tau}$. They are related among each other by

$$
\begin{aligned}
& \vartheta_{1}(z, q)=-i e^{i z+\frac{1}{4} i \pi \tau} \vartheta_{4}\left(z+\frac{1}{2} \pi \tau, q\right), \\
& \vartheta_{2}(z, q)=\vartheta_{1}\left(z+\frac{1}{2} \pi, q\right), \\
& \vartheta_{3}(z, q)=\vartheta_{4}\left(z+\frac{1}{2} \pi, q\right) .
\end{aligned}
$$


The $\vartheta$-functions can be written as infinite products as follows

$$
\begin{aligned}
& \vartheta_{1}(z, q)=2 q^{\frac{1}{4}} \sin z G \prod_{n=1}^{\infty}\left(1-2 q^{2 n} \cos 2 z+q^{4 n}\right), \\
& \vartheta_{2}(z, q)=2 q^{\frac{1}{4}} \cos z G \prod_{n=1}^{\infty}\left(1+2 q^{2 n} \cos 2 z+q^{4 n}\right), \\
& \vartheta_{3}(z, q)=G \prod_{n=1}^{\infty}\left(1+2 q^{2 n-1} \cos 2 z+q^{4 n-2}\right), \\
& \vartheta_{4}(z, q)=G \prod_{n=1}^{\infty}\left(1-2 q^{2 n-1} \cos 2 z+q^{4 n-2}\right),
\end{aligned}
$$

where $G$ is given by

$$
G=\prod_{n=1}^{\infty}\left(1-q^{2 n}\right) .
$$

We further define $\phi(z, q)$ by $\vartheta_{1}(z, q)=\sin z \phi(z, q)$.

\section{Periodicities of $\vartheta$-functions}

The $\vartheta$-functions are quasi doubly-periodic in $z$ with period $\pi$ and $\pi \tau$. Under transformation of $z$ by a period they pick up the factors

\begin{tabular}{|c|c|c|c|c|}
\hline & $\vartheta_{1}(z, q)$ & $\vartheta_{2}(z, q)$ & $\vartheta_{3}(z, q)$ & $\vartheta_{4}(z, q)$ \\
\hline$\pi$ & -1 & -1 & 1 & 1 \\
\hline$\pi \tau$ & $-N$ & $N$ & $N$ & $-N$ \\
\hline
\end{tabular}

where $N=q^{-1} e^{-2 i z}$.

By considering the definitions of the $\vartheta$-functions we can see at once that $\vartheta_{1}(z, q)$ is an odd function in $z$ whereas all other $\vartheta$-functions are even in $z$.

The zeros of $\vartheta$-functions

If $\vartheta_{i}(z, q)$ has a zero at $z_{0}$ the quasi-periodicity implies it has also zeros at

$$
z_{0}+m \pi+n \pi \tau, \quad n, m \in \mathbb{Z} .
$$

It can be shown that in the fundamental domain with corners $\{0, \pi, \pi \tau, \pi+\pi \tau\}$ the zeros are given by

\begin{tabular}{|c|c|c|c|}
\hline$\vartheta_{1}(z, q)$ & $\vartheta_{2}(z, q)$ & $\vartheta_{3}(z, q)$ & $\vartheta_{4}(z, q)$ \\
\hline 0 & $\frac{1}{2} \pi$ & $\frac{1}{2} \pi+\frac{1}{2} \pi \tau$ & $\frac{1}{2} \pi \tau$ \\
\hline
\end{tabular}

\section{$\vartheta$-function identities}

$\vartheta$-functions are connected via a vast amount of identities of which we will only list very few here. Two remarkable identities are

$$
\vartheta_{1}^{\prime}(0, q)=\vartheta_{2}(0, q) \vartheta_{3}(0, q) \vartheta_{4}(0, q)
$$


where the prime denotes a differentiation with respect to $z$ and the well known identity

$$
\vartheta_{2}(0, q)^{4}+\vartheta_{4}(0, q)^{4}=\vartheta_{3}(0, q)^{4},
$$

which can be generalised to

$$
\vartheta_{2}(z, q)^{4}+\vartheta_{4}(z, q)^{4}=\vartheta_{1}(z, q)^{4}+\vartheta_{3}(z, q)^{4} .
$$

From formula (B.4) we can deduce the interesting product identity

$$
1=\prod_{n=1}^{\infty}\left(1+q^{2 n}\right) \prod_{n=1}^{\infty}\left(1+q^{2 n-1}\right) \prod_{n=1}^{\infty}\left(1-q^{2 n-1}\right)
$$

by writing out the $\vartheta$-functions as products.

\section{Modular transformations}

It is sometimes convenient to write the $\vartheta$-functions as functions of $\tau$ and $z$, rather than as functions of $q$ and $z$. In order to distinguish this from the previous definition of the $\vartheta$-functions we use the symbol $\vartheta_{i}(z \mid \tau)$ in that case. In particular, this is convenient when discussing the modular transformation properties of the $\vartheta$-functions:

\begin{tabular}{|c|c|c|c|c|}
\hline & $\vartheta_{1}(z \mid \tau)$ & $\vartheta_{2}(z \mid \tau)$ & $\vartheta_{3}(z \mid \tau)$ & $\vartheta_{4}(z \mid \tau)$ \\
\hline$\tau^{\prime}=\tau+1$ & $e^{-\frac{\pi i}{4}} \vartheta_{1}\left(z \mid \tau^{\prime}\right)$ & $e^{-\frac{\pi i}{4}} \vartheta_{2}\left(z \mid \tau^{\prime}\right)$ & $\vartheta_{4}\left(z \mid \tau^{\prime}\right)$ & $\vartheta_{3}\left(z \mid \tau^{\prime}\right)$ \\
\hline$\tau^{\prime}=\frac{-1}{\tau}$ & $-i A \vartheta_{1}\left(z \tau^{\prime} \mid \tau^{\prime}\right)$ & $A \vartheta_{4}\left(z \tau^{\prime} \mid \tau^{\prime}\right)$ & $A \vartheta_{3}\left(z \tau^{\prime} \mid \tau^{\prime}\right)$ & $A \vartheta_{2}\left(z \tau^{\prime} \mid \tau^{\prime}\right)$ \\
\hline
\end{tabular}

where $A=(-i \tau)^{-1 / 2} e^{\frac{i z^{2} \tau^{\prime}}{\pi}}$.

\section{Proof of the $\vartheta$-function identity}

We want to prove that

$$
\mathcal{A}=q^{-\frac{1}{4}} \frac{\prod_{n=1}^{\infty}\left(1+2 \cos (\Delta) q^{2 n-1}+q^{4 n-2}\right)}{\prod_{n=1}^{\infty}\left(1-2 \cos (\Delta) q^{2 n}+q^{4 n}\right)}
$$

is equal to

$$
\mathcal{A}_{\phi_{1}, \phi_{2}}=q^{-\frac{1}{4}} \prod_{n=1}^{\infty} \frac{\left(1-q^{2 n-1}\right)^{2}}{\left(1-q^{2 n}\right)^{2}}\left[1+2 \sum_{n=1}^{\infty}[\cos (n \Delta)-\cos ((n-1) \Delta)] \frac{q^{2 n-1}}{1+q^{2 n-1}}\right] .
$$

The former expression can be easily written in terms of $\vartheta$-functions,

$$
\mathcal{A}=2 \sin \left(\frac{\Delta}{2}\right) \frac{\vartheta_{3}\left(\frac{\Delta}{2}, q\right)}{\vartheta_{1}\left(\frac{\Delta}{2}, q\right)} .
$$

Similarly, we can easily write the prefactor of (C.2) in this manner

$$
\mathcal{A}_{\phi_{1}, \phi_{2}}=2 \frac{\vartheta_{3}(0, q)}{\phi(0, q)}\left[1+2 \sum_{n=1}^{\infty}[\cos (n \Delta)-\cos ((n-1) \Delta)] \frac{q^{2 n-1}}{1+q^{2 n-1}}\right],
$$


where $\vartheta_{1}(z, q)=\sin (z) \phi(z, q)$. To show the equality of the two expressions we will treat them as analytic functions in the first variable and show that their periodicities, zeros and poles are identical. So, what we want to prove is

$$
\begin{aligned}
\frac{\vartheta_{3}(z, q)}{\vartheta_{1}(z, q)} & =\frac{1}{\sin (z)} \frac{\vartheta_{3}(0, q)}{\phi(0, q)}\left[1+2 \sum_{n=1}^{\infty}[\cos (2 n z)-\cos (2(n-1) z)] \frac{q^{2 n-1}}{1+q^{2 n-1}}\right] \\
& =\frac{1}{\sin (z)} \frac{\vartheta_{3}(0, q)}{\phi(0, q)} \Sigma
\end{aligned}
$$

where we have divided both sides by $2 \sin (z)$, and where $\Sigma$ is the expression in brackets

$$
\Sigma=1+2 \sum_{n=1}^{\infty}[\cos (2 n z)-\cos (2(n-1) z)] \frac{q^{2 n-1}}{1+q^{2 n-1}} .
$$

The zeros of the left hand side of (C.5) are given by $\frac{\pi}{2}+\frac{\pi \tau}{2}$. If we insert this value for $z$ into the expression for $\Sigma$ we get

$$
1+\sum_{n=1}^{\infty}(-1)^{n}\left[q^{n}+q^{n-1}\right]=0
$$

which shows that $\frac{\pi}{2}+\frac{\pi \tau}{2}$ is indeed a zero of the right hand side. In order to show that the right hand side of equation (C.5) does not have any additional zeros we use the trigonometric identity

$$
\cos (2 n z)-\cos (2(n-1) z)=-2 \sin (z) \sin ((2 n-1) z)
$$

to rewrite $\Sigma$ as

$$
\begin{aligned}
\Sigma & =2 \sin (z)\left[\frac{1}{2 \sin (z)}-2 \sum_{n=1}^{\infty} \sin (2 n-1) z \frac{q^{2 n-1}}{1+q^{2 n-1}}\right] \\
& =2 \sin (z)\left[-2 i \sum_{n=1}^{\infty} \frac{q^{\frac{2 n-1}{2}}}{1+q^{2 n-1}} \cos \left((2 n-1)\left(z-\frac{\pi \tau}{2}\right)\right)\right]
\end{aligned}
$$

where we have used the expansion

$$
\frac{1}{\sin z}=e^{i z} \frac{-2 i}{1-e^{2 i z}}=-2 i \sum_{n=1}^{\infty} e^{i(2 n-1) z} .
$$

It is then manifest that $\Sigma$ does not have any other zeros in the fundamental cell.

Given (C.9) it is now also obvious that both sides of (C.5) have a single pole at $z=0$, and that the residue is the same. Thus we have shown that both sides of (C.5) have the same poles and zeros (as a function of $z$ ).

Finally, it remains to prove that both sides of (C.5) have the same periodic properties in $z$. The $\vartheta$-functions are doubly periodic with periods $\pi$ and $\pi+\pi \tau$ (see appendix (B). From (C.5) it is manifest that the right hand side changes sign under $z \mapsto z+\pi$, as well as under $z \mapsto-z$. The behaviour of the left hand side is identical, as can be seen from the periodicities of the $\vartheta$-functions, as well as by direct inspection. Under the shift $z \mapsto z+\pi \tau$ the left hand side changes sign. For the right hand side we observe that the bracket in $\Sigma$ (C.10) transforms 
under $z \mapsto z+\pi \tau$ in the same way as under $z \mapsto-z$. Since the factor of $\sin (z)$ in $\Sigma($ (C.10) is cancelled in (C.5) it then follows that the right hand side therefore also changes sign under $z \mapsto z+\pi \tau$. Thus we have shown that the two sides of (C.5) have the same periodicity properties. Since their poles and zeros agree, it then follows that they are indeed the same function.

\section{References}

[1] J. Polchinski, Combinatorics of boundaries in string theory, Phys. Rev. D50 (1994) 6041, hep-th/9407031.

[2] D. Friedan, The space of conformal boundary conditions for the $c=1$ Gaussian model, unpublished note (1999).

[3] M.R. Gaberdiel, A. Recknagel and G.M.T. Watts, The conformal boundary states for SU(2) at level 1, Nucl. Phys. B626 (2002) 344, hep-th/0108102.

[4] M.R. Gaberdiel, A. Recknagel, Conformal boundary states for free bosons and fermions, JHEP 11 (2001) 016, hep-th/0108238.

[5] R.A. Janik, Exceptional boundary states at $c=1$, Nucl. Phys. B618 (2001) 675, hep-th/0109021.

[6] L.-S. Tseng, A note on $c=1$ Virasoro boundary states and asymmetric shift orbifolds, JHEP 04 (2002) 051, hep-th/0201254.

[7] T. Banks, L.J. Dixon, D. Friedan and E.J. Martinec, Phenomenology and conformal field theory or can string theory predict the weak mixing angle?, Nucl. Phys. B299 (1988) 613.

[8] H. Ooguri, Y. Oz and Z. Yin, D-branes on Calabi-Yau spaces and their mirrors, Nucl. Phys. B477 (1996) 407, hep-th/9606112

[9] A. Recknagel and V. Schomerus, D-branes in Gepner models, Nucl. Phys. B531 (1998) 185, hep-th/9712186.

[10] K. Hori, A. Iqbal and C. Vafa, D-branes and mirror symmetry, hep-th/0005247.

[11] U. Lindström and M. Zabzine, $N=2$ boundary conditions for non-linear sigma models and Landau-Ginzburg models, JHEP 02 (2003) 006, hep-th/0209098

[12] C. Albertsson, U. Lindström and M. Zabzine, Superconformal boundary conditions for the WZW model, JHEP 05 (2003) 050, hep-th/0304013.

[13] J. Maldacena, G.W. Moore and N. Seiberg, Geometrical interpretation of D-branes in gauged WZW models, JHEP 07 (2001) 046, hep-th/0105038.

[14] S. Fredenhagen and V. Schomerus, D-branes in coset models, JHEP 02 (2002) 005, hep-th/0111189.

[15] S.E. Parkhomenko, Free field construction of D-branes in $N=2$ superconformal minimal models, Nucl. Phys. B671 (2003) 325, hep-th/0301070. 
[16] S. Fredenhagen, Organizing boundary RG flows, Nucl. Phys. B660 (2003) 436, hep-th/0301229.

[17] T. Eguchi and Y. Sugawara, Modular bootstrap for boundary $N=2$ Liouville theory, JHEP 01 (2004) 025, hep-th/0311141.

[18] C. Ahn, M. Stanishkov and M. Yamamoto, One-point functions of $N=2$ super-Liouville theory with boundary, Nucl. Phys. B683 (2004) 177, hep-th/0311169.

[19] C. Ahn and M. Yamamoto, Boundary action of $N=2$ super-Liouville theory, Phys. Rev. D69 (2004) 026007, hep-th/0310046.

[20] M. Ademollo, L. Brink, A. D'Adda, R. D'Auria, E. Napolitano, S. Sciuto, E. Del Giudice, P. Di Vecchia, S. Ferrara, F. Gliozzi, R. Musto and R. Pettorino, Supersymmetric strings and color confinement, Phys. Lett. B62 (1976) 105.

[21] M. Dörrzapf, Superconformal field theories and their representations, Ph.D. thesis, University of Cambridge, 1995.

[22] H. Klemm, Embedding diagrams of the $N=2$ superconformal algebra under spectral flow, hep-th/0306073, to appear in Int. J. Mod. Phys A.

[23] A. Schwimmer and N. Seiberg, Comments on the $N=2,3,4$ superconformal algebras in two dimensions, Phys. Lett. B184 (1987) 191.

[24] W. Lerche, C. Vafa and N.P. Warner, Chiral rings in N=2 superconformal theories, Nucl. Phys. B324 (1989) 427.

[25] N. Ishibashi, The boundary and crosscap states in conformal field theories, Mod. Phys. Lett. A4 (1989) 251.

[26] J.L. Cardy, Boundary conditions, fusion rules and the Verlinde formula, Nucl. Phys. B324 (1989) 581.

[27] C. Bachas, Lectures on D-branes, (1998), hep-th/9806199.

[28] C. Bachas and M. Porrati, Pair creation of open strings in an electric field, Phys. Lett. B296 (1992) 77, hep-th/9209032

[29] A. Abouelsaood, C.G. Callan Jr., C.R. Nappi and S.A. Yost, Open strings in background gauge fields, Nucl. Phys. B280 (1987) 599.

[30] P. Di Vecchia and A. Liccardo, D-branes in string theory. I, (1999), hep-th/9912161.

[31] P. Di Vecchia and A. Liccardo, D-branes in string theory. II, (1999), hep-th/9912275.

[32] M.R. Gaberdiel, Lectures on non-BPS Dirichlet branes, Class. Quant. Grav. 17 (2000) 3483 , hep-th/0005029.

[33] B. Craps and M.R. Gaberdiel, Discrete torsion orbifolds and D-branes II, JHEP 04 (2001) 013, hep-th/0101143.

[34] A. Erdélyi, W. Magnus, F. Oberhettinger and G.F. Tricomi, Higher Transcendental Functions (The Bateman Manuscript), vol. II, McGraw-Hill, 1953. 
[35] E.T. Whittaker and G.N. Watson, A Course of Modern Analysis, Cambridge University Press, 1958. 